\title{
A Streamlined Technique to Examine Cell Monolayers by means of Correlative Light and Transmission Electron Microscopy
}

\author{
W.G. Janssen ${ }^{1}$, H.H. Hanson ${ }^{1}$ and B.L. Armbruster ${ }^{2}$ \\ 1. Department of Neuroscience, Mount Sinai School of Medicine, One Gustave Levy Place, New York, \\ NY 10029 \\ 2. Hitachi High Technologies America, Inc., Nanotechnology Systems Division, 5960 Inglewood Drive, \\ Pleasanton, CA 94588
}

Correlative light/electron microscopy (CLEM) combines the power of light microscopy to search large areas for fluorescently-tagged live cells/regions of interest and transmission electron microscopy (TEM) to image the same specimen at high magnification for ultrastructural characterization. CLEM methods are essential to observe dynamic processes such as organelle biogenesis and intracellular trafficking and then investigate the same cell regions with the resolution of the TEM. Although previously described techniques can be technically demanding, a simplified protocol would facilitate CLEM in nonspecialized laboratories.

A CLEM protocol is described for embedding cell monolayers on gridded glass bottom live imaging dishes after imaging transfected GFP-tagged proteins by means of laser scanning confocal microscopy (LSCM) [1]. This streamlined, low cost method allows rapid EM processing without equipment beyond what is standard for LSCM and TEM labs. LSCM imaging and specimen preparation for TEM are completed in the same culture dish without transfer of samples. Complete separation of the resin block with embedded cells from the glass surface is accomplished by low heating on a hot plate instead of freezing [2] or dissolving the glass with acid [3].

For LSCM, Mouse oligodendrocyte progenitors were isolated from P6-P8 C57B16 mice and cultured as previously described [4]. Cells were plated on uncoated gridded glass bottom dishes (MatTek Corp., Ashland MA, USA). Cells transfected with GFP were fixed with $2 \%$ paraformaldehyde and 2\% glutaraldehyde/phosphate buffer and imaged on an inverted Zeiss LSM 780 LSCM equipped with a stage for mounting the live imaging dishes. GFP was excited by $488 \mathrm{~nm}$ wavelength argon laser light and its emission detected through a long-pass $505 \mathrm{~nm}$ filter. Both DIC and GFP LSCM z-stack images were taken using a $63 \mathrm{X}$ objective (Plan-Apo 1.4NA, oil).

For TEM, the cells were fixed with $1 \%$ osmium tetroxide and $1.5 \%$ potassium ferracyanide in $0.1 \mathrm{M}$ cacodylate buffer prior to an ethanol dehydration series and infiltration with Embed 812. The approximate location of the cell of interest was notched into the glass coverslip. An open ended embedding capsule with a $1 \times 1 \mathrm{~cm}$ face was placed on the coverslip, filled with resin and polymerized in a vacuum oven. The block was separated from the dish with careful heating, retaining all the cells and an imprint of the etched grid. Thin serial sections were stained with uranyl acetate and lead citrate and the grids were imaged on a Hitachi HT7700 TEM at $80 \mathrm{kV}$.

The CLEM protocol described here relies on DIC imaging and prominent GFP labeling within individual cells to be used as landmarks for light and electron microscopy. In Figure 1 several GFPpositive profiles are visible at low magnification and one cell is selected for serial section TEM analysis. The method is extremely useful for GFP-labeled organelles in culture and could conceivably be applied 
to structure/function studies of organelle trafficking or biogenesis by means of mutant tagged cDNA constructs.

\section{References:}

[1] HH Hanson et al, Microsc. Microanal. 16 (2010) 747.

[2] M Razi and SA Tooze, Methods Enzymol. 452 (2009) 261.

[3] M Grabenbauer, Methods Cell Biology 111 (2012) 117.

[4] JD Cahoy et al, Journal of Neurosci. 28, (2008) 264.
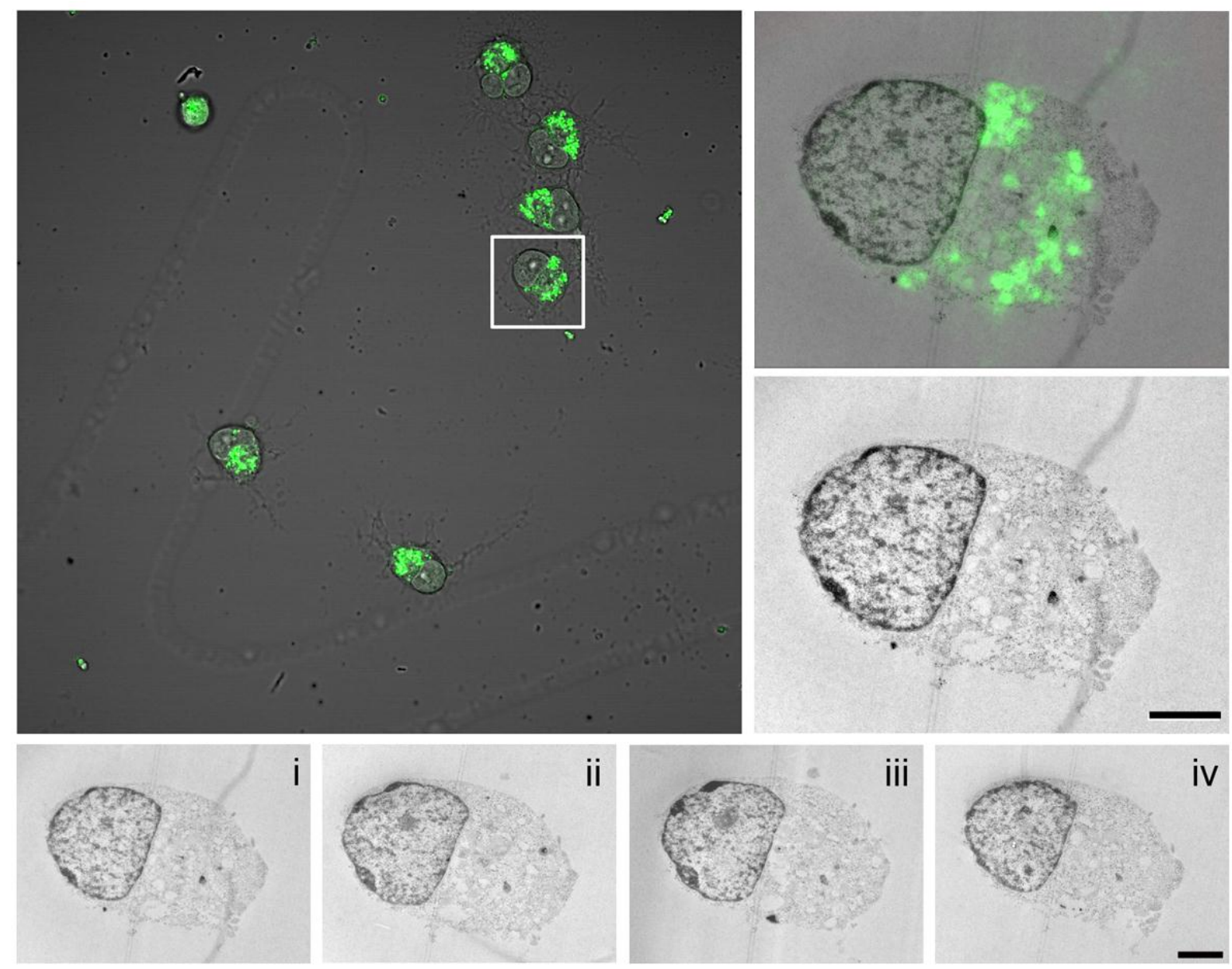

Figure 1. Low power DIC/GFP image showing the cells of interest with respect to the alphanumeric grid etched on the coverslip. The boxed region was selected for high magnification DIC/GFP and TEM correlative imaging (upper right shows the individual cell imaged for TEM with GFP overlay, and directly below is the TEM sectioned cell minus overlay). Individual serial sections i-iv of one cell are displayed in the bottom row. Scale bars equal $2 \mu \mathrm{m}$. In practice all cells within this plane of view are imaged, embedded and serially sectioned for analysis. 